University of Wollongong

Research Online

Faculty of Engineering and Information

Faculty of Engineering and Information

Sciences - Papers: Part B

Sciences

2019

The effects of vertical and horizontal sources on heat transfer and entropy generation in an inclined triangular enclosure filled with non-Newtonian fluid and subjected to magnetic field

Zhixiong $\mathrm{Li}$

University of Wollongong, lizhixio@uow.edu.au

Amin Shahsavar

Kavian Niazi

Abdullah Al-Rashed

Pouyan Talebizadehsardari

Follow this and additional works at: https://ro.uow.edu.au/eispapers1

Part of the Engineering Commons, and the Science and Technology Studies Commons

Research Online is the open access institutional repository for the University of Wollongong. For further information contact the UOW Library: research-pubs@uow.edu.au 


\title{
The effects of vertical and horizontal sources on heat transfer and entropy generation in an inclined triangular enclosure filled with non-Newtonian fluid and subjected to magnetic field
}

\author{
Abstract \\ 2019 Elsevier B.V. Natural convection and entropy generation of a power-law non-Newtonian fluid in a \\ tilted triangular enclosure subjected to a magnetic field was investigated. A part of the enclosure's right or \\ left wall is at a high temperature while the top wall is cold. The remaining walls are insulated. The results \\ indicate that when the hot wall is at the left wall and the Rayleigh number is increased from 103 to 105 , \\ the heat transfer rate of the shear-thinning fluid goes up 1.5 times and its entropy generation rate rises $>2$ \\ fold. For the Newtonian fluid, these changes mean an increase of $71 \%$ in heat transfer and a surge of $80 \%$ \\ in entropy generation. With the increase of Rayleigh number, Bejan number diminishes. A higher \\ Hartmann number results in a lower average Nusselt number and entropy generation rate and the rise in \\ the Bejan number in the considered enclosure. \\ Disciplines \\ Engineering | Science and Technology Studies \\ Publication Details \\ Li, Z., Shahsavar, A., Niazi, K., Al-Rashed, A. \& Talebizadehsardari, P. (2019). The effects of vertical and \\ horizontal sources on heat transfer and entropy generation in an inclined triangular enclosure filled with \\ non-Newtonian fluid and subjected to magnetic field. Powder Technology,
}

This journal article is available at Research Online: https://ro.uow.edu.au/eispapers1/3789 
The Effects of Vertical and Horizontal source on Heat Transfer and Entropy

Generation in an Inclined Triangular Enclosure Filled with Non-Newtonian Fluid and Subjected to Magnetic Field

Zhixiong Li ${ }^{1}$, Amin Shahsavar ${ }^{2}$, Kavian Niazi ${ }^{2}$, Abdullah AAA Al-Rashed ${ }^{3}$, and Pouyan Talebizadehsardari ${ }^{4 *}$

1. School of Mechanical, Materials, Mechatronic and Biomedical Engineering, University of Wollongong, NSW 2522, Australia

2. Department of Mechanical Engineering, Kermanshah University of Technology, Kermanshah, Iran

3. Department of Automotive and Marine Engineering Technology, College of Technological Studies, The Public Authority for Applied Education and Training, Kuwait

4. Faculty of Engineering, University of Nottingham, Nottingham, United Kingdom

*Corresponding author

E-mail addresses: pouyan.talebizadehsardari@ nottingham.ac.uk (Pouyan Talebizadehsardari)

\begin{abstract}
Natural convection and entropy generation of a power-law non-Newtonian fluid in a tilted triangular enclosure subjected to a magnetic field was investigated. A part of the enclosure's right or left wall is at a high temperature while the top wall is cold. The remaining walls are insulated. The results indicate that when the hot wall is at the left wall and the Rayleigh number is increased from $10^{3}$ to $10^{5}$, the heat transfer rate of the shear-thinning fluid goes up 1.5 times and its entropy generation rate rises more than 2 fold. For the Newtonian fluid, these changes mean an increase of $71 \%$ in heat transfer and a surge of $80 \%$ in entropy generation. With the increase of Rayleigh number, Bejan number diminishes. A higher Hartmann number results in a lower average Nusselt number and entropy generation rate and the rise in the Bejan number in the considered enclosure.
\end{abstract}


Keywords: Entropy generation; Non-Newtonian fluid; Power-law model; Triangular enclosure; Magnetic field; Natural convection.

\begin{tabular}{|c|c|c|c|}
\hline \multicolumn{4}{|c|}{ Nomenclatures } \\
\hline$B$ & magnetic field strength & $u$ & Velocity in $\mathrm{x}$ direction \\
\hline$B e$ & Bejan number & $v$ & Velocity in y direction \\
\hline$C$ & Lattice speed & $x, y$ & Cartesian coordinates \\
\hline$C_{p}$ & Specific heat & Greek letter & \\
\hline$F$ & Body forces & $\sigma$ & Electrical conductivity \\
\hline$f$ & Density distribution functions & $\phi$ & Relaxation time \\
\hline$f_{e q}$ & Equilibrium density distribution functions & $\tau$ & Shear stress \\
\hline$g$ & Internal energy distribution functions & $\zeta$ & Discrete particle speeds \\
\hline$g_{e q}$ & Equilibrium internal energy distribution functions & $\Delta x$ & Lattice spacing \\
\hline $\mathrm{g}$ & Gravity acceleration & $\Delta t$ & Time increment \\
\hline $\mathrm{H}$ & Non-dimensional length & $\alpha$ & Thermal diffusivity \\
\hline $\mathrm{Ha}$ & Hartmann number & $\rho$ & Density \\
\hline$K$ & Consistency coefficient & $\mu$ & Dynamic viscosity \\
\hline 1 & length & $\psi$ & Stream function value \\
\hline$L_{1}$ & Heat source length & & \\
\hline $\mathrm{L}$ & Heat source non-dimensional length & Subscripts & \\
\hline$n$ & Power-law index & Gen & Generation \\
\hline$N u$ & Nusselt number & GenT & Thermal Generation \\
\hline$P$ & Pressure & ave & Average \\
\hline $\operatorname{Pr}$ & Prandtl number & C & Cold \\
\hline$R a$ & Rayleigh number & $H$ & Hot \\
\hline$S$ & entropy & $x, y$ & Cartesian coordinates \\
\hline$t$ & Time & $\alpha$ & Node number \\
\hline$T$ & Temperature & & \\
\hline
\end{tabular}

\section{Introduction}

More research works have been conducted on enclosed cavities in recent years. By examining different geometries and designing various problems, researchers have investigated the subject of natural convection in different applications [1-10]. Owing to 
the extensive use of enclosed cavities in various industries related to solar power, food, defense, aerospace and others, numerous researchers have employed different fluids to study the subjects of flow and temperature fields in enclosures. Non-Newtonian fluids are one type of fluid which has been used as working fluid by some researchers in their studies [11-20]. Many of the fluids utilized in various industries are Non-Newtonian; examples include different types of dyes, sauces, pastes, glues, and some oils [21-33]. In these fluids, the relationship between shear stress and strain rate cannot be defined by Newton's stress formula. In this regard, equations such as the power-law model, Bingham, etc. can be used to express the relationship between stress and strain and to calculate the amount of shear stress. Alsabery et al. [34] employed the Finite Element Method to investigate the natural convective heat transfer in a power-law NonNewtonian fluid inside a trapezoidal cavity. Using a non-Newtonian nano-fluid, Kefayati et al. [35] explored the subject of natural convection in a square. They insulated the top and bottom and kept the sidewalls at constant high and low temperatures. They used the power-law model and a power-law index range of 0.6-1.0. Mishra et al. [36] studied an enclosure with two inner circular obstacles. For analyzing their Non-Newtonian fluid, they applied the power-law model and used a power-law index range of 0.2-2.

In many applications for enclosed cavities, these enclosures are subjected to a magnetic field; which can be produced by applying an electric current. The magnetic field effect on devise containing non-Newtonian or Newtonian fluids has also been investigated by several researchers [37-41]. Kefayati [42] applied the Lattice Boltzmann Method (LBM) to study the horizontal magnetic field effect on the flow field of a non-Newtonian fluid in a square cavity. They varied the power-law index from 0.5 to 1.5 and found out that a higher Hartmann number results in a heat transfer reduction. Using the LBM, Kefayati and Tang [37] explored the natural convection in a power-law non-Newtonian fluid 
within a square. They placed two circular sources in the middle of the enclosure and studied their effect. They also applied a magnetic field to the enclosure and realized that the presence of a magnetic field has an adverse effect on the rate of heat transfer. Applying the FDLBM, Aghakhani et al. [43] investigated the natural convection in a power-law non-Newtonian fluid within a C-shaped cavity. They exposed the enclosure to a magnetic field and evaluated the effects of Rayleigh number, Hartmann number, aspect ratio, and power-law index on heat transfer rate. They discovered that the rate of heat transfer diminishes by increasing the power-law index. The heat transfer rate also goes up with the rise of Rayleigh number and the reduction of Hartmann number.

The increased utilization of energy by man, especially in recent years, and the dwindling fossil fuel resources have prompted the researchers to try to cut down on energy consumption by improving the efficiencies of various devices and equipment. The higher consumption of fossil fuels has caused a variety of environmental problems around the globe, including the global warming, climatic changes, melting of natural glaciers, air pollution, and many other calamities. In dealing with the issue of energy loss in various appliances, researchers have examined the amount of entropy generated in these devices. Based on the second law of thermodynamics, entropy shows the amount of irreversibility generated in a process; and by analyzing it, the energy loss of that process can be determined [44, 45]. So, many researchers have investigated the amount of entropy generation in various enclosures [46-55]. Siavashi et al. [56] studied the natural convection in a power-law non-Newtonian fluid inside an enclosure between two circles. They also examined the amount of entropy generation. Using the LBM, Kefayati [39] investigated the rate of natural convective heat transfer in non-Newtonian fluid. He applied a horizontal magnetic field to the considered enclosure and also analyzed the amount of entropy generation. He found out that both the heat transfer and entropy 
generation go up with the increase of Rayleigh number. Raizah et al. [57] studied the natural convection heat transfer in a power-law non-Newtonian fluid. By tilting the enclosure at different angles, they evaluated the amount of heat transfer rate and entropy generation.

Non-Newtonian fluids are utilized in a variety of industrial applications, and many of the fluids used in engineering processes are non-Newtonian. One of the models employed in determining the shear stress of non-Newtonian fluids is the power-law model. The use of non-Newtonian fluids in enclosures and cavities, because of its application in various industries such as food, military, etc., has become the subject of interest for many researchers. The magnetic field effect on the flow field within an enclosure is an important issue to investigate. It is also necessary to explore the amount of entropy generation in an enclosure in order to find out its efficiency and energy loss. Most of the enclosures studied by researchers have rectangular and square shapes, and cavities of other shapes have been investigated less frequently. The main reason for this is that it is rather difficult to apply a mesh to a non-rectangular enclosure. So in the present work, the natural convective heat transfer and the entropy generation of a non-Newtonian fluid within a triangular enclosure are studied. This enclosure is tilted with respect to the horizon and it is also subjected to a horizontal magnetic field. The power-law model is employed to model the non-Newtonian fluid's shear stress. A portion of one of the enclosure walls is at a high temperature. The subject matter is studied by varying the size and location of this hot region.

The main novelty of this work is to investigate the effect of the length of a heated wall located on two triangular chambers filled with a non-Newtonian fluid on the heat transfer and entropy generation behaviour of the fluid in the presence of a magnetic field. Also, 
studying the effect of the chamber angle with the above-mentioned conditions can help to find better physical conditions to enhance the heat transfer.

Finally, the effects of influential parameters such as Rayleigh number, Hartmann number, power-law index, hot wall length and enclosure tilt angle on heat transfer rate, entropy generation and Bejan number are investigated.

\section{Defining the problem}

The geometry of the problem includes a 2-D triangular enclosure of sides $\mathrm{H}$, which is tilted at angle $\gamma$ relative to the horizon. The top wall is at the temperature of $\mathrm{T}_{\mathrm{c}}$. A portion of the left wall (Case a) or the right wall (Case b) with length $L$ is at temperature $T_{h}$. The other sections of the walls have been insulated. A magnetic field of strength $\mathrm{B}_{0}$ is also applied to the enclosure in the horizontal direction. The enclosure has been filled with a non-Newtonian fluid. And the power-law model has been used to model the shear stress of the fluid.

\section{Governing equations}

The main equations governing the behavior of fluid flow, in non-dimensional form, are as follows. These equations have been written by assuming a constant laminar flow and an incompressible fluid. The Boussinesq approximation has been used to produce the buoyancy force in the enclosure and the loss of viscosity has been ignored [43].

$$
\begin{aligned}
& \frac{\partial \mathrm{U}}{\partial \mathrm{X}}+\frac{\partial \mathrm{V}}{\partial \mathrm{Y}}=0 \\
& \mathrm{U} \frac{\partial \mathrm{U}}{\partial \mathrm{X}}+\mathrm{V} \frac{\partial \mathrm{U}}{\partial \mathrm{Y}}=-\frac{\partial \mathrm{P}}{\partial \mathrm{X}}+\frac{\operatorname{Pr}}{\sqrt{\mathrm{Ra}}}\left(\frac{\partial \tau_{\mathrm{XX}}}{\partial \mathrm{X}}+\frac{\partial \tau_{\mathrm{XY}}}{\partial \mathrm{Y}}\right)-\frac{\mathrm{PrHa}^{2}}{\sqrt{\mathrm{Ra}}} \mathrm{U}+\operatorname{Pr} \theta \operatorname{Sin} \gamma \\
& \mathrm{U} \frac{\partial \mathrm{V}}{\partial \mathrm{X}}+\mathrm{V} \frac{\partial \mathrm{V}}{\partial \mathrm{Y}}=-\frac{1}{\rho} \frac{\partial \mathrm{P}}{\partial \mathrm{Y}}+\frac{\operatorname{Pr}}{\sqrt{\mathrm{Ra}}}\left(\frac{\partial \tau_{\mathrm{XX}}}{\partial \mathrm{X}}+\frac{\partial \tau_{\mathrm{XY}}}{\partial \mathrm{Y}}\right)+\operatorname{Pr} \theta \operatorname{Cos} \gamma
\end{aligned}
$$




$$
\begin{aligned}
& \left(\mathrm{U} \frac{\partial \theta}{\partial \mathrm{X}}+\mathrm{V} \frac{\partial \theta}{\partial \mathrm{Y}}\right)=\frac{1}{\sqrt{\mathrm{Ra}}}\left(\frac{\partial^{2} \theta}{\partial \mathrm{X}^{2}}+\frac{\partial^{2} \theta}{\partial \mathrm{Y}^{2}}\right) \\
& \tau_{\mathrm{IJ}}=2 \mu_{\mathrm{a}}^{*} \mathrm{D}_{\mathrm{IJ}}=\mu_{\mathrm{a}}^{*}\left(\frac{\partial \mathrm{U}_{\mathrm{i}}}{\partial \mathrm{X}_{\mathrm{j}}}+\frac{\partial \mathrm{V}_{\mathrm{j}}}{\partial \mathrm{Y}_{\mathrm{i}}}\right) \\
& \mu_{\mathrm{a}}^{*}=\mathrm{K}\left\{2\left[\left(\frac{\partial \mathrm{U}}{\partial \mathrm{X}}\right)^{2}+\left(\frac{\partial \mathrm{V}}{\partial \mathrm{Y}}\right)^{2}\right]+\left(\frac{\partial \mathrm{V}}{\partial \mathrm{X}}+\frac{\partial \mathrm{U}}{\partial \mathrm{Y}}\right)^{2}\right\}^{\frac{(\mathrm{n}-1)}{2}} \\
& S_{\text {gen }}=\underbrace{\left(\left(\frac{\partial \theta}{\partial \mathrm{x}}\right)^{2}+\left(\frac{\partial \theta}{\partial \mathrm{y}}\right)^{2}\right)}_{\text {Thermal }}+\underbrace{\zeta\left\{2\left[\left(\frac{\partial U}{\partial \mathrm{X}}\right)^{2}+\left(\frac{\partial \mathrm{V}}{\partial \mathrm{Y}}\right)^{2}\right]+\left(\frac{\partial \mathrm{U}}{\partial \mathrm{Y}}+\frac{\partial \mathrm{V}}{\partial \mathrm{X}}\right)^{2}\right\}}_{\text {Fluid friction }}+\underbrace{\zeta \text { Ha }^{2}(U)^{2}}_{\text {magnetic }} \\
& \Psi(X, Y)=\int U d Y+\Psi_{0}
\end{aligned}
$$

In Eq. $6, \mathrm{~K}$ and $\mathrm{n}$ denote the constants of the exponential function model. $\mathrm{K}$ is the coefficient and $n$ is the index of the exponential function. For shear thickening fluids, $n$ $>1$; and for shear thinning fluids, $\mathrm{n}<1$; while $\mathrm{n}=1$ means Newtonian fluids.

Many of the fluids used to simulate non-Newtonian fluid flows have been simulated using the power-law function. This is due to the similar behavior of many non-Newtonian fluids to this function, which has caused this model to attract more attention among researchers. Many of the nanofluids have been cited by researchers as having a power-law behavior. This model is capable of simulating non-Newtonian dilatant and pseudoplastic fluids. This model is also used in this study for non-Newtonian fluid analysis.

The following parameters is used to dimensionless the governing equations and the boundary conditions. The Rayleigh number (Ra), Hartmann number (Ha), Bejan number (Be), entropy parameter $(\zeta)$, and the Prandtl number (Pr) have been defined in these parameters.

$$
\begin{aligned}
& X=\frac{x}{l} \quad, Y=\frac{y}{l}, U=\frac{u}{\left(\frac{\alpha}{l}\right) R a^{0.5}}, V=\frac{v}{\left(\frac{\alpha}{l}\right) R a^{0.5}} P=\frac{p}{\rho\left(\frac{\alpha}{l}\right)^{2} R a}, \theta=\frac{T-T_{c}}{T_{H}-T_{c}}, L= \\
& \frac{l_{1}}{l}, H=\frac{l}{l} \\
& \operatorname{Pr}=\frac{\mu_{a}}{\rho \alpha}, R a=\frac{\rho g \beta l^{3}\left(T_{h}-T_{c}\right)}{\mu_{a} \alpha_{f}}, H a=B_{0} l \sqrt{\frac{\sigma}{\mu_{a}}}, \zeta=\frac{\mu_{n f} T_{0}}{k_{f}}\left(\frac{\alpha_{f}}{l\left(T_{h}-T_{C}\right)}\right)^{2}
\end{aligned}
$$




$$
\tau_{I J}=\frac{\tau_{i j}}{\mu_{a}}, \alpha=\frac{k}{\rho C_{P}}
$$

The governing non-dimensional boundary conditions for the problem have been listed in

Table 1 and Table 2 for case (a) and case (b), respectively.

\section{Numerical method}

LBM has become an accurate method for simulating computational fluid dynamic problems and it has been used more frequently in recent years for such purposes. So in the present paper, LBM is used to simulate the flow field and temperature distribution; as will be described in the following sections. Some of the advantages of this method include its ease of implementation and its capability of performing parallel computations $[58,59]$.

In this research, the D2Q9 Lattice-Boltzmann model has been used for simulation (see Fig. 2). In LBM, the distribution function expresses the probability of particles existing at a definite time and in a specific region. The relevant distribution function must satisfy the continuity, momentum and energy equations at a macroscopic scale. However, the Lattice Boltzmann equations can be extracted in Finite Difference form from the Boltzmann transfer function. The subject of discussion in the Lattice-Boltzmann equation (LBE) is this particle distribution function. For solving the equations in the present research, a new approach called the Finite Difference LBM (FDLBM) is employed. In fact, one of the most important issues is the ability to simulate non-Newtonian fluids; since in these fluids, local viscosity changes with velocity gradient. Therefore, due to the non-constant relaxation time, the LBM cannot be employed to solve a whole lot of problems [50-62]. By using the discretization technique, Fu et al. [63-65] presented new equations for equilibrium distribution function, instead of Maxwell's equations. They have actually assumed a variable equilibrium distribution function and a constant 
relaxation time; and by doing so, they have eliminated the limitation of the LatticeBoltzmann method. Moreover, in this approach, the pressure is a variable parameter and, contrary to the conventional LBM, it is not obtained directly from density. In fact, they have applied a Finite Difference Lattice-Boltzmann Method to solve the problem. This method is capable of extracting the shear stress from the base equation of LatticeBoltzmann method for flow fields. Additionally, different boundary conditions can be applied in the FDLBM, without encountering the problems and limitations that exist in the conventional LBM.

The function that expresses the probability of the presence of particles (moving with specific speed $\xi$ ) at location $r$ and at time $t$. According to the LBE, the governing equation for distribution function $f$ is given as:

$$
\frac{\partial \mathrm{f}_{\alpha}}{\partial \mathrm{t}}+\xi_{\alpha} \cdot \nabla \mathrm{f}_{\alpha}=-\frac{1}{\varepsilon \phi}\left(\mathrm{f}_{\alpha}-\mathrm{f}_{\alpha}^{\mathrm{eq}}\right)
$$

Also, $\varepsilon$ is a small parameter, which is taken to be equal to the time step $(\varepsilon=\Delta \mathrm{t})$.

If Eq. 10 is discretized and the relaxation time is taken to be $\phi=1$, the diffusion part of the equation can be written as Eq. 11:

$$
\frac{\partial \mathrm{f}_{\alpha}}{\partial \mathrm{t}}+\xi_{\alpha} \cdot \nabla_{\mathrm{X}} \mathrm{f}_{\alpha}=0
$$

Eq. 17 is obtained by applying the Lax-Vanderhoff method. The impact part of the equation, in discrete form, can be written as follows:

$$
\frac{\partial \mathrm{f}_{\alpha}}{\partial \mathrm{t}}=-\frac{1}{\varepsilon \phi}\left(\mathrm{f}_{\alpha}(\mathrm{X}, \mathrm{t})-\mathrm{f}_{\alpha}^{\mathrm{eq}}(\mathrm{X}, \mathrm{t})\right)
$$

The diffusion part of the equation is used as appropriate condition for solving the impact part; and by employing the Euler method and choosing $\varepsilon=\mathrm{f}$ and $\phi=1$, Eq. 10 can be written as Eq. 13:

$$
\frac{\mathrm{f}_{\alpha}(\mathrm{x}, \mathrm{t}+\Delta \mathrm{t})-\mathrm{f}_{\alpha}(\mathrm{X}, \mathrm{t})}{\Delta \mathrm{t}}=-\frac{1}{\varepsilon \phi}\left(\mathrm{f}_{\alpha}(\mathrm{X}, \mathrm{t})-\mathrm{f}_{\alpha}^{\mathrm{eq}}(\mathrm{X}, \mathrm{t})\right)
$$


Thus,

$$
\left(f_{\alpha}(X, t+\Delta t)=f_{\alpha}^{e q}(X, t)\right)
$$

For the density distribution function, the Chapman-Anscock expansion has been used as

Eq. 15:

$$
\begin{aligned}
& f_{\alpha}=f_{\alpha}^{e q}+\varepsilon f_{\alpha}^{(1)}+\varepsilon^{2} f_{\alpha}^{(2)}+O\left(\varepsilon^{3}\right) \\
& \sum_{\alpha=0}^{8} f_{\alpha}^{(n)}=\sum_{\alpha=0}^{8} f_{\alpha}^{(n)} \xi_{\alpha x}=\sum_{\alpha=0}^{8} f_{\alpha}^{(n)} \xi_{\alpha y}=0, \quad n \geq 1 \\
& \sum_{\alpha=0}^{8} f_{\alpha}^{e q}=\rho=\text { constant } \\
& \sum_{\alpha=0}^{8} f_{\alpha}^{e q} \xi_{\alpha x}=\rho u \\
& \sum_{\alpha=0}^{8} f_{\alpha}^{e q} \xi_{\alpha y}=\rho v \\
& \sum_{\alpha=0}^{8} \mathrm{f}_{\alpha}^{\mathrm{eq}} \xi_{\alpha x} \xi_{\alpha y}=\rho v u-\tau_{x y} \mathrm{f}_{\alpha}^{\text {eq }} \xi_{\alpha y}^{2}=\rho v^{2}+p-\tau_{y y} \\
& \sum_{\alpha=0}^{e q} \xi_{\alpha x}^{2}=\rho u^{2}+p-\tau_{x x}
\end{aligned}
$$

With the help of the above equations, the Navier-Stokes equations can be derived from the Lattice-Boltzmann equation:

$$
\frac{\partial(\rho u)}{\partial x}+\frac{\partial(\rho v)}{\partial y}=0
$$


$\frac{\partial \rho u^{2}}{\partial x}+\frac{\partial \rho v u}{\partial y}=-\frac{\partial p}{\partial x}+\left(\frac{\partial \tau_{x x}}{\partial x}+\frac{\partial \tau_{x y}}{\partial y}\right)+O(\varepsilon)$

$\frac{\partial \rho v^{2}}{\partial y}+\frac{\partial \rho v u}{\partial x}=-\frac{\partial p}{\partial y}+\left(\frac{\partial \tau_{y y}}{\partial y}+\frac{\partial \tau_{x y}}{\partial x}\right)+O(\varepsilon)$

where $\rho$ is the density that is assumed to be constant. For the viscosity, the second-order Chapman-Enskog expansion term, which contains the relaxation time, is used. Therefore, this model allows viscosity to be a function of the shear rate. The effort to develop the LBM is for problems in simulating non-Newtonian fluids. Unfortunately, the relaxation time destabilizes the numerical simulations, especially in the case of non-Newtonian fluid flow, when the shear rate drops to zero. Therefore, relaxation time must be controlled to ensure solution convergence. The small relaxation time means that from the point of view of analysis, scale and rank of the viscosity terms relative to other terms is relatively insignificant and causes this problem not to be realized. To overcome this problem, Chapman-Enskog expansion is used to recover Navier-Stokes equations. The LBM finite difference method is used for modeling non-Newtonian fluids.

The viscosity can be easily considered as a variable for non-Newtonian fluids. If $\mathrm{f}_{\alpha}^{\text {eq }}$ is appropriate has an appropriate value, by using Eqs. 16 through 22, the solution of Eq. 9 will, in fact, be the solution of Navier-Stokes equation (2-3) with an error of $O(\varepsilon)$. A second-degree polynomial series $\left(\xi_{\alpha}\right)$ has been assumed as the first suggestion initial value for the variable of $\mathrm{f}_{\alpha}^{\mathrm{eq}}$ for microflows, which does not follow the older viewpoint. Therefore, $\mathrm{f}_{\alpha}^{\mathrm{eq}}$ is totally different from the Maxwell-Boltzmann distribution function obtained from the second-degree or third-degree trimmed expansion with $U$ and $\xi_{\alpha}$.

$$
\begin{gathered}
f_{\alpha}^{e q}=A_{\alpha}+\left(\xi_{\alpha}\right)_{X} A X_{\alpha}+\left(\xi_{\alpha}\right)_{Y} A Y_{\alpha}+\left(\xi_{\alpha}\right)_{X}^{2} B X X_{\alpha}+\left(\xi_{\alpha}\right)_{Y}^{2} B Y Y_{\alpha} \\
+\left(\xi_{\alpha}\right)_{X}\left(\xi_{\alpha}\right)_{Y} B X Y_{\alpha}
\end{gathered}
$$

Parameter $\xi_{\alpha}$ depends on the selected model. The model used for discretizing the Boltzmann equation on a lattice is generally displayed as $D_{n} Q_{m}$; where $n$ is indicative of 
dimension and $\mathrm{m}$ is the number of permitted directions for the movement of particles. The $\mathrm{D}_{2} \mathrm{Q}_{9}$ model is the most common 2-D model. This type of lattice is shown in Fig. 2. The values of $\xi_{\alpha}$ are expressed as Eq. 27. In this equation, $c$ is the speed of virtual particles on the lattice; which should be determined with the consideration of other parameters [65].

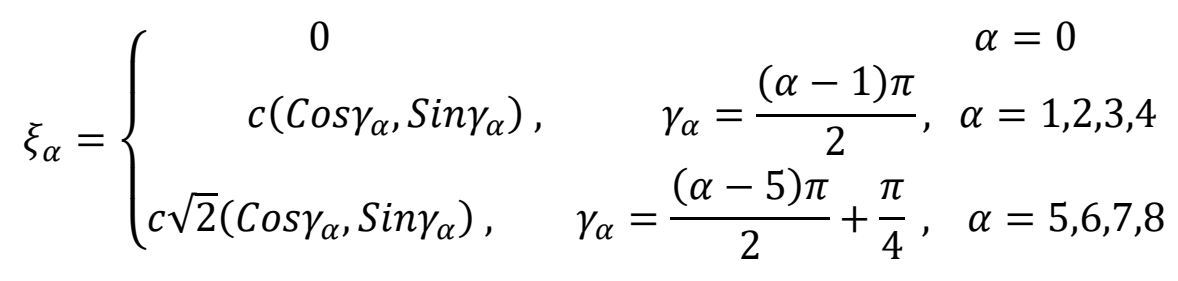

The other parameters in the microscopic velocity distribution equation are as follows:

$$
\begin{aligned}
& A_{0}=\rho-\frac{2 \rho}{c^{2}}-\frac{\rho|u|^{2}}{c^{2}}+\frac{\tau_{X X}+\tau_{Y Y}}{c^{2}}, \quad A_{1}=A_{2}=0 \\
& A x_{1}=\frac{\rho U}{2 c^{2}}, A X_{2}=0 \\
& A Y_{1}=\frac{\rho V}{2 c^{2}}, A Y_{2}=0 \\
& B X X_{1}=\frac{1}{2 c^{4}}\left(P+\rho U^{2}-\tau_{X X}\right), \quad B X X_{2}=0 \\
& B Y Y_{1}=\frac{1}{2 c^{4}}\left(P+\rho V^{2}-\tau_{Y Y}\right), \quad B Y Y_{2}=0 \\
& B X Y_{2}=\frac{1}{4 c^{2}}\left(\rho V U-\tau_{X Y}\right), \quad B X Y_{1}=0, \quad I, J=X, Y
\end{aligned}
$$

The considered coefficients in Eq. 29 are defined as follows:

$$
\begin{array}{ll}
A_{1}=A_{3}=A_{5}=A_{7} & A_{2}=A_{4}=A_{6}=A_{8} \\
A X_{1}=A X_{3}=A X_{5}=A X_{7} & A X_{2}=A X_{4}=A X_{6}=A X_{8} \\
A Y_{1}=A Y_{3}=A Y_{5}=A Y_{7} & A Y_{2}=A Y_{4}=A Y_{6}=A Y_{8} \\
B X X_{1}=B X X_{3}=B X X_{5}=B X X_{7} & B X X_{2}=B X X_{4}=B X X_{6}=B X X_{8} \\
B Y Y_{1}=B Y Y_{3}=B Y Y_{5}=B Y Y_{7} & B Y Y_{2}=B Y Y_{4}=B Y Y_{6}=B Y Y_{8} \\
& B X Y_{2}=B X Y_{4}=B X Y_{6}=B X Y_{8}
\end{array}
$$




$$
\tau_{I J}=\frac{\operatorname{Pr} \mu_{\alpha}}{K \sqrt{R a}}\left(\frac{\partial U}{\partial Y}+\frac{\partial V}{\partial X}\right)
$$

The magnetic field and buoyancy force terms in the momentum equation are added to the diffusion parts of Eqs. 30 .

$$
\begin{aligned}
& f_{\alpha}=A_{\alpha}+\left(\xi_{\alpha}\right)_{X} A X_{\alpha}+\left(\xi_{\alpha}\right)_{Y} A Y_{\alpha} \\
& A_{0}=A_{1}=A_{2}=0 \\
& A X_{1}=\frac{\operatorname{Pr} \theta \operatorname{Sin} \gamma-\frac{P r H a^{2}}{\sqrt{R a}} U}{2 c^{2}}, \quad A X_{2}=0 \\
& A Y_{1}=\frac{\operatorname{Pr} \theta \operatorname{Cos} \gamma}{2 c^{2}}, \quad A Y_{2}=0
\end{aligned}
$$

By disregarding the loss of viscosity, the Lattice-Boltzmann energy equation is obtained as Eq. 31:

$$
\begin{aligned}
& \frac{\partial g_{\alpha}}{\partial t}+\xi_{\alpha} \cdot \nabla g_{\alpha}=-\frac{1}{\varepsilon \phi}\left(g_{\alpha}-g_{\alpha}^{e q}\right) \\
& \frac{g_{\alpha}(x, t+\Delta t)-g_{\alpha}(x, t)}{\Delta t}=-\frac{1}{\varepsilon \phi}\left(g(x, t)-g_{\alpha}^{e q}(x, t)\right)
\end{aligned}
$$

$\mathrm{g}_{\alpha}$ is discretized in the direction of velocity. The equilibrium and the thermal energy distribution functions have been expressed as follows:

$$
\begin{aligned}
& g_{\alpha}^{e q}=A_{\alpha}+\left(\xi_{\alpha}\right)_{X} A X_{\alpha}+\left(\xi_{\alpha}\right)_{Y} A Y_{\alpha} \\
& A_{0}=\theta, \quad A_{1}=A_{2}=0 \\
& A X_{1}=\frac{U \theta-\frac{1}{\sqrt{R a}} \frac{\partial \theta}{\partial X}}{2 c^{2}}, \quad A X_{2}=0 \\
& A Y_{1}=\frac{V \theta-\frac{1}{\sqrt{R a}} \frac{\partial \theta}{\partial Y}}{2 c^{2}}, \quad A Y_{2}=0
\end{aligned}
$$

\section{Boundary conditions}


The boundary condition applied to the enclosure is the mirror reflection boundary condition. This condition is equivalent to the no-slip-at-wall boundary condition. To apply the boundary condition, the flow distribution functions, after the impact of flow with a wall, are reflected along the flow's movement path. Fig. 3 shows the manner of applying the mirror reflection boundary condition. The distribution functions indicated by a dash line are unknown functions. This boundary condition is used on triangle chord and other sides.

Considering the definition of mirror reflection condition, the unknown functions of Fig. 3 are computed by means of Eq. 33, which can also be generalized for other boundaries.

$$
\begin{aligned}
& f_{2}=f_{4} \\
& f_{5}=f_{7} \\
& f_{6}=f_{8}
\end{aligned}
$$

The constant-temperature boundary conditions have been presented in Eqs. 34.

Hot wall

$$
\begin{array}{ll}
g_{1}=\left(T_{h}\left(w_{1}+w_{3}\right)\right)-g_{3} & g_{3}=\left(T_{c}\left(w_{1}+w_{3}\right)\right)-g_{1} \\
g_{5}=\left(T_{h}\left(w_{5}+w_{7}\right)\right)-g_{7} & g_{7}=\left(T_{c}\left(w_{5}+w_{7}\right)\right)-g_{5} \\
g_{8}=\left(T_{h}\left(w_{8}+w_{6}\right)\right)-g_{6} & g_{6}=\left(T_{c}\left(w_{8}+w_{6}\right)\right)-g_{8}
\end{array}
$$

Cold wall

To measure the amount of heat transfer, the local and the average Nusselt numbers on hot walls are computed by means of Eqs. 35 and 36, respectively.

$$
\begin{aligned}
& \mathrm{Nu}=\left(-\frac{\partial \theta}{\partial \mathrm{n}}\right), \quad n=X, Y \quad, \quad \mathrm{X}=0, \quad, \quad Y=0 \\
& \mathrm{Nu}_{\text {Ave }}=\int_{0}^{\mathrm{L}} \mathrm{Nud} n \quad n=X, Y
\end{aligned}
$$

Total entropy can be obtained by integration over the entire solution boundary. 


$$
S_{g e n}=\int S_{g e n} d \Omega=\iint_{0}^{1} S_{g e n} d X d Y
$$

Also to evaluate the degree of irreversibility, the Bejan number and the average Bejan number are respectively obtained from Eqs. 38 and 39.

$$
\begin{aligned}
& B e=\frac{S_{\text {gen,T }}}{S_{\text {Total }}} \\
& B_{\text {Ave }}=\iint_{0}^{1} B e d X d Y
\end{aligned}
$$

\section{The mesh-independency and the validation of results}

To evaluate the independency of the solution from the number of mesh points, 6 different mesh configurations have been used according to Table 3 for cases (a) and (b) and for $\mathrm{Ra}=10^{5}, \mathrm{Ha}=20$ and two exponential function indices of $\mathrm{n}=0.6$ and $\mathrm{n}=1.4$. Table 3 shows the values of average Nusselt number on the hot wall $\left(\mathrm{Nu}_{\mathrm{Ave}}\right)$, average entropy and maximum flow function $\left(\Psi_{\max }\right)$ for these 6 mesh configurations. Since the difference between the results of mesh sizes $140 \times 140,160 \times 160$ and $180 \times 180$ in Table 3 is small, mesh size $160 \times 160$ or 19600 nodes was deemed as appropriate for use in the computations performed in this work. In this case, the size of each mesh is 0.00625 .

To confirm the accuracy of the computer program and to validate the average $\mathrm{Nu}$ values obtained for different exponential function indexes, the values related to flow and heat transfer inside a square enclosure with constant-temperature vertical walls and insulated horizontal walls containing an exponential function model non-Newtonian fluid have been compared in Table 4 with the results of Khezzar [66] and Huilgol and Kefayati [67]. at Rayleigh numbers of $10^{4}$ and $10^{5}$. Also, the average $\mathrm{Nu}$ obtained for the mentioned enclosure under the effect of the magnetic field have been compared with the results of Kefayati [42] in Fig. 4. 


\section{Analysis of results}

\subsection{Variations of Rayleigh number and power-law index}

Fig. 5 illustrates the contours of flow lines in cases (a) and (b) for different $R a$ and $n$ for $H a=20, L=0.3, \gamma=45^{\circ}$. In both cases, a large vortex can be seen within the enclosure. Also, the maximum value of flow function, which shows the vortex strength, has been written at the center of each vortex. The creation of a vortex in the enclosure is due to the buoyancy force. The buoyancy force arises from the difference in fluid densities, which itself is due to the temperature difference within the enclosure. The difference between wall temperatures causes a temperature difference in the fluid and eventually leads to the formation of the vortex inside the enclosure. The higher this temperature difference, the greater the buoyancy force and the stronger the vortex formed inside the enclosure. The rise in wall temperature difference means the increase of $R a$. So in all the considered cases, the formed vortex in the enclosure becomes stronger for a higher $R a$. A magnetic field along the horizontal axis is applied to the enclosure. This causes the fluid to move away from the left wall of the enclosure and approach the right wall. This is why the vortex formed in case (b) is stronger than that in case (a); because in case (a), fluid moves away from the hot wall and thus the vortex is weakened, but in case (b), the magnetic field drives the fluid toward the hot wall and creates more contact between them, thereby enhancing the buoyancy force. Also, the rotation directions of vortexes in cases (a) and (b) are opposite to each other. In case (a) the formed vortex rotates counterclockwise, while in case (b) it has a clockwise rotation. The vortex rotation direction depends on the direction of gravity and the magnetic field applied. The shifting of hot walls also has a great influence on the direction of vortex rotation. For a higher $n$, 
the apparent viscosity of the fluid increases as well, which causes the fluid to circulate more slowly in the enclosure. At high $R a$, vortex strength is reduced with the increase of $n$. At low $R a$, vortex velocity is very low, and the variation of $n$ has no effect on vortex velocity.

Fig. 6 shows the contours of isothermal lines in cases (a) and (b) for different $R a$ and $n$ for $H a=20, L=0.3, \gamma=45^{\circ}$. The concentration of isothermal lines is higher where there is a greater temperature difference. Hence, it is observed that in all the examined cases, the concentration of isothermal lines is higher over a hot wall than at any other region. So the temperature gradient and the amount of heat transfer are also greater in these regions. At low $R a$, the isothermal lines enjoy a certain orderliness and regularity. However, with the increase of $R a$, the isothermal lines become irregular and disordered. The regularity or the disorderliness of isothermal lines indicates the mechanism of heat transfer. The regular isothermal lines portend a stronger conduction heat transfer and the disorderly isothermal lines mean a stronger convection heat transfer. So a stronger conduction heat transfer results at low $R a$ and a stronger convection heat transfer ensues at high $R a$, and the underlying cause is vortex strength. At low $R a$, vortex strength is negligible and, thus, the enclosure fluid is almost stationary; but at high $R a$, vortex strength is considerable and there is movement in the enclosure fluid. Also, with the rise of $R a$, the isothermal lines near the hot and cold walls get more compacted; which means the increase of temperature gradient and heat transfer rate in these regions. The elevated temperature gradient is caused by the faster movement of the vortex and the higher fluid velocity near the constant-temperature walls. The concentration location of isothermal lines changes by altering the position of the hot wall. Because of a stronger vortex in case (b), the concentration of isothermal lines is slightly greater in case (b) than in case (a). With the increase of $n$, the concentration of isothermal lines is severely reduced and they 
become more regular and less curly. This means that, with the rise of $n$, the conduction heat transfer mechanism becomes stronger and more dominant against the natural convection mechanism. The reason for these changes with the rise of $n$ is the reduction of vortex velocity with the increase of fluid's apparent viscosity; which leads to the reduction of fluid velocity and temperature gradient in the enclosure.

Fig. 7 displays the contours of generated entropy lines in cases (a) and (b) for different $R a$ and $n$ for $H a=20, L=0.3, \gamma=45^{\circ}$. A high entropy generation is shown in the regions where the temperature gradient is high. There is also a concentration of entropy generation lines in these regions. This shows that the increase of temperature gradient boosts the generation of entropy in the enclosure. The reason for this can be sought in the constituent components of the total generated entropy. Total entropy comprises three components of thermal, fluid friction and magnetic field, which, respectively, depend on temperature gradient, velocity gradient, and fluid velocity and $\mathrm{Ha}$. By raising $R a$, fluid velocity and the temperature gradient in the enclosure are increased, thereby boosting the entropy generation. The fluid's apparent viscosity goes up with a higher $n$. As the apparent viscosity of fluid surges in the flow field and temperature field, the vortex velocity and the temperature gradient are reduced, thereby diminishing the fluid friction and thermal entropies, respectively. This leads to the decline of total entropy generation. Also, the entropy field shows that the concentration of generated entropy lines goes down with the rise of $n$. By shifting the position of the hot wall in the enclosure, the location of generated entropy lines changes and is always positioned over the hot wall and indicates that the entropy generation rate is greater in the regions that have a hightemperature gradient.

The local $N u$ on the hot wall of enclosure is plotted in Fig 8 for case a (different $n$ for $R a=10^{5}, H a=20, L=0.3, \gamma=45^{\circ}$ ) and case $\mathrm{b}$ (different $R a$ and $H a=20, n=$ 
1.4, $\left.L=0.3, \gamma=45^{\circ}\right)$. According to the figure, at a specific $n$, with the increase of $\mathrm{x}$, $N u$ goes up first and then starts to decrease and, as we approach the end of the hot wall, it rises again. The low $\mathrm{v}$ at the beginning section of the hot wall is due to the small space there, which inhibits the infiltration of fluid into this region and reduces the contact of the cold fluid with the hot wall. The local $N u$ diminishes with the rise of $n$. The reason for this is that, with the increase of $n$, the apparent fluid viscosity goes up, and with the increase of fluid viscosity, vortex velocity diminishes under similar conditions. With the decline of fluid flow velocity in the enclosure, the temperature gradient decreases near the cold wall. This is due to the increase of thermal boundary layer thickness according to the increase of dynamic boundary layer thickness. The reduction of temperature gradient lowers the rate of heat transfer over the enclosure wall. At low $R a$, the local $N u$ does not change with the rise of $R a$. However, with the further increase of $R a$, the heat transfer rate rises at the beginning section of the hot wall and falls at the end section. Since these graphs have been plotted for $n=1.4$, the fluid velocity is hardly affected by $R a$. At low $R a$, fluid velocity does not change significantly with the rise of $R a$ and, therefore, the heat transfer rate remains unchanged. With the further increment of $R a$, fluid velocity in the enclosure escalates, more fluid flows into the lower section, which has little space for fluid infiltration, and consequently, the heat transfer rate goes up in this region. Also, with more fluid infiltration in this region, fluid can move along a longer section of the hot wall and get warmer. So, when the fluid reaches the midsection of the hot wall, it will have a higher temperature; this will reduce the temperature gradient, and the local $\mathrm{Nu}$ will diminish as well.

Fig. 9 illustrates the average $N u$ on the hot wall of enclosure in case a (dash line) and case $\mathrm{b}$ (dash-dot line) for different $R a$ and $n$ for $H a=20, L=0.3, \gamma=45^{\circ}$. This figure shows that at low $R a$, the rate of heat transfer in case (b) is greater than that in case 
(a). The reason for this is the direction of the magnetic field, which directs the fluid toward the right wall of the enclosure. So in the case in which the hot wall is on the right enclosure wall, the fluid is in contact with the hot wall for a longer period and gets warmer; thus, the heat transfer rate increases. At low $R a$, in both cases, the average $N u$ changes very little with $n$, and $N u$ remains constant. The reason for this is that at low $R a$, fluid velocity within the enclosure is very small and the fluctuations of fluid's apparent viscosity have no effect on vortex velocity. Therefore, since the fluid velocity does not vary, the heat transfer rate also remains constant. However, at high $R a$, heat transfer rate diminishes considerably with the rise of $n$. This is due to the reduction of vortex velocity with the increase of fluid's apparent viscosity in the enclosure. As the apparent viscosity goes up, shear stress increases and fluid velocity diminishes, thereby lowering the rate of heat transfer within the enclosure. With the reduction of fluid velocity, convective heat transfer is weakened and conductive heat transfer is reinforced; so the total amount of heat transfer is reduced as well. At high $R a$, case (a) is more sensitive to the variations $n$ and, at low $n$, it has a larger $N u$ and, at high $n$, it has a smaller average $\mathrm{Nu}$ relative to the case (b). The diagrams indicate that in the case of high apparent viscosity and low $R a$, case (b) produces a higher heat transfer rate and in the case of low apparent viscosity and high $R a$, case (a) yields a better heat transfer rate.

Fig. 10 displays the total generated entropies in case a (dash line) and case b (dash-dot line) for different $R a$ and $n$ for $H a=20, L=0.3, \gamma=45^{\circ}$. Here we can also see that at low $R a$, the total generated entropy varies very little with the changes of $n$. The reason is that fluid velocity does not vary with $n$ at low $R a$. With the rise of $R a$, temperature and velocity gradients go up; thus, the amounts of thermal and friction entropies also enhance, thereby boosting the total generated entropy. At high $R a$, the total generated entropy diminishes with a higher $n$. The underlying cause is the reduction of vortex 
velocity inside the enclosure with the increase of fluid's apparent viscosity. With the reduction of vortex velocity, temperature and velocity gradients in the enclosure also go down. This diminishes the thermal entropy and fluid friction entropy, respectively, and leads to the reduction of total generated entropy. At a high $R a$ and low apparent viscosity, because of the elevated fluid velocity in case (a), the total generated entropy in this case is greater than the other cases. In case (a), considering the existing flow field, a strong vortex is formed inside the enclosure, which helps raise the temperature and velocity gradients and eventually increase the amount of generated entropy.

Fig. 11 shows the plotted values of $B e$ in case a (dash line) and case $\mathrm{b}$ (dash-dot line) for different $R a$ and $n$ for $H a=20, L=0.3, \gamma=45^{\circ} . B e$ indicates the share of thermal entropy in the total generated entropy. The higher $B e$, the larger the share of thermal entropy in the total generated entropy. $B e$ is higher at low $R a$ and is lower at high $R a$. The reason is that at low $R a$, flow velocity in the enclosure is negligible and temperature gradient, which leads to the generation of thermal entropy, has a large share in the total generated entropy. With the rise of $R a$ and fluid velocity in the enclosure, the velocity gradient and thus the fluid friction entropy increase, thereby reducing the share of thermal entropy in the total generated entropy. As a result, $B e$ diminishes with the increase of $R a$. This occurs in both cases. In general, with the increase of $n$, fluid's apparent viscosity goes up, and because of the rise of shear stress in fluid flow, vortex velocity diminishes. Thus, the values of velocity gradient and fluid friction entropy dwindle as well. This increases the thermal entropy contribution in the total generated entropy and leads to the rise of $B e$.

\subsection{Changing the Hartmann number and the length of enclosure's hot wall}


Fig. 12 demonstrates the contours of flow lines in case (a) and case (b) for various $\mathrm{Ha}$, three different hot wall lengths for $R a=10^{5}, n=1, \gamma=45^{\circ}$. By exposing the enclosure to a magnetic field, a force called the Lorentz force is applied to the enclosure. This force drives the fluid in the direction of the magnetic field and tries to keep the fluid steady. Hence, the Lorentz force counteracts the buoyancy force which moves the fluid in the enclosure. So the presence of the magnetic field leads to the reduction of buoyancy force. The buoyancy force is the cause of vortex formation in the enclosure, and with its decline, the strength of formed vortexes is reduced. Therefore, it is observed that in both cases, vortex strength diminishes with the increase of $\mathrm{Ha}$ (i.e., the boosting of Lorentz force). With the rise of $\mathrm{Ha}$, the formed vortex moves away from the left enclosure wall and is squeezed toward the right wall. In case (a), this causes the fluid to have less contact with the hot wall and, therefore, less heat transfer takes place between the hot wall and fluid. In case (b), the fluid remains near the hot wall and is not displaced considerably. This lowers the temperature difference in the enclosure and reduces the vortex velocity. By increasing the hot wall length, fluid is exposed to high temperatures along a longer section of the wall and becomes warmer. Thus, the temperature difference and density difference in the enclosure are increased. The rise of density difference fortifies the buoyancy force and adds to vortex strength. As vortex strength is boosted, faster vortex forms in the enclosure, which occupies most of the fluid regions and penetrates the sharp corners of the enclosure more easily.

Fig. 13 shows the contours of isothermal lines in both cases (a) and (b) for various $\mathrm{Ha}$, three different hot wall lengths for $R a=10^{5}, n=1, \gamma=45^{\circ}$. With the increase of $\mathrm{Ha}$, the isothermal lines become more regular and less disordered. Regular and uniform temperature lines indicate a greater conduction heat transfer, and irregular and disrupted temperature lines characterize a stronger convection heat transfer. Therefore in both 
cases, with the rise of $\mathrm{Ha}$, conduction heat transfer is fortified and convection heat transfer is weakened. This is due to the reduction of fluid velocity in the enclosure with the increase of $\mathrm{Ha}$. Also, as $\mathrm{Ha}$ rises, the isothermal lines near the hot wall become less compact, which means the decline of temperature gradient and heat transfer rate. Again, this is due to the reduction of flow velocity and, thus, the increase in the thickness of the dynamic boundary layer. As the thickness of the dynamic boundary layer increases, so does the thickness of the thermal boundary layer and, consequently, the temperature gradient goes down. In both cases, it is observed that the temperature gradient over the hot wall is higher than the other regions. By making the hot wall longer, fluid is more exposed to a wall of high temperature and gets warmer. This increases the vortex velocity with regards to the flow field. Moreover, with the rise of fluid temperature in the enclosure, the temperature gradient near the cold wall also increases and causes a surge of heat transfer rate. This can be deduced from the considerable increase in the concentration of isothermal lines near the cold wall. By adding to the length of the thermal wall, not only the conductive heat transfer is boosted by getting the hot wall closer to the cold wall, but also the convective heat transfer is fortified by increasing the vortex velocity; and eventually, the temperature gradient in the vicinity of the cold wall is raised.

Fig. 14 illustrates the plotted contours of generated entropy lines in cases (a) and (b) for various $H a$, three different hot wall lengths and for $R a=10^{5}, n=1, \gamma=45^{\circ}$. It is observed that the concentration of generated entropy lines diminishes with the increase of $\mathrm{Ha}$. The reason is the decrease in the temperature and velocity gradients with the rise of $\mathrm{Ha}$, according to temperature and flow fields. The decline of temperature and velocity gradients diminishes the thermal entropy and the fluid friction entropy, respectively, and lowers the total entropy accordingly. The rise of $\mathrm{Ha}$ does not affect the component of 
magnetic field entropy; because the magnetic field entropy includes both the $H a$ and the fluid flow velocity, and these two have an inverse relationship with each other. As $\mathrm{Ha}$ rises, fluid velocity falls, and so the magnetic field entropy remains almost unchanged. Conversely, the magnetic field entropy has a much lower value compared to thermal and friction entropies; so the total generated entropy is more affected by the values of thermal entropy and fluid friction entropy and much less by the magnetic field entropy. In both cases, there are a large number of generated entropy lines over the hot wall; which is indicative of a high-temperature gradient in that region. By adding to the length of the hot wall, the concentration of entropy lines, especially on the cold wall, increases. This is due to the rise of temperature gradient in that region. As it was mentioned, with the increase in the length of the hot wall, the temperature gradient near the hot wall goes up, thereby boosting the generation of thermal entropy and increasing the value of total generated entropy in that region.

Fig. 15(a) displays the non-dimensional horizontal velocities and Fig. 15(b) the nondimensional temperatures at line $\mathrm{X}=0.5$ for different $\mathrm{Ha}$ for $\mathrm{Ra}=10^{5}, n=1, L=0.3$, $\gamma=45^{\circ}$ in case (a). According to the figure, at a specific $\mathrm{Ha}$, fluid velocity has two maximum positive and negative values. This is due to the existence of a vortex in the enclosure. Fluid velocity has negative values in the upper section of the enclosure and positive values in the lower section. This indicates the presence of a counterclockwise rotating vortex in the enclosure. The velocity values diminish with the increase of $H a$. The reason, as was previously mentioned, is the Lorentz force counteracting the buoyancy force; which leads to a decline of vortex velocity in the enclosure. The figure also shows the drop of non-dimensional temperature with the rise of $\mathrm{Ha}$. This is caused by the decrease of fluid velocity, which reduces the temperature gradient near the constant-temperature wall and diminishes the heat transfer rate. Thus, temperature 
variations within the enclosure are also lowered. At low $H a$, curvatures appear in the temperature profile, which indicates fluid movement in the enclosure. With the rise of $\mathrm{Ha}$ and the diminishing of vortex velocity, these curves become smoother and the temperature profile assumes a more even shape, and it outlines the temperatures from the vicinity of the hot wall to cold wall in a regular manner.

As Fig. 16 illustrates, at most of the considered hot wall lengths, the non-dimensional temperature profile has two maximum negative and positive values; which is also indicative of vortex generation in the enclosure. However, as the hot wall gets larger, the negative and positive values switch places, and the negative velocity now becomes positive. This shows a change in the direction of vortex rotation. When the hightemperature wall is small and up to a size of 0.3 , a counterclockwise rotating vortex is present in the enclosure and, as the hot wall becomes bigger, it turns into a clockwise rotating vortex. With the increase in the hot wall length, velocity values become greater and they also have more maximums. This is due to the rise of vortex velocity with the increase of hot wall length. The figure also shows that the increase of hot wall length leads to the rise of non-dimensional temperature at the beginning section of the diagram; so that at a hot wall size of over 0.5 , it reaches a value of 1.0. The reason is that the hot wall gets closer to the beginning part of the diagram and causes the temperature to go up in that region. Moreover, the fluid temperature in the enclosure is raised with the prolonged contact of the fluid with the hot wall; which can be seen clearly in the graph of non-dimensional temperature.

The average $\mathrm{Nu}$ on the hot wall is plotted in Fig. 17 in cases (a) and (b) for different $\mathrm{Ha}$ and hot wall lengths for $R a=10^{5}, n=1, \gamma=45^{\circ}$. Both figures show a decline in heat transfer rate with the increase of $\mathrm{Ha}$. This arises from the reduction of fluid velocity with the rise of $\mathrm{Ha}$, for reasons already mentioned. Also, the increase in hot wall length has 
caused the average $N u$ to go up. There are two reasons for this: first, with the increase of hot wall length, fluid velocity escalates due to the surge in buoyancy force; this boosts the convective heat transfer and also raises the temperature gradient in the enclosure, which increases the heat transfer rate. Second, with the enclosure's hot wall getting closer to the cold wall, the existing conductive heat transfer capacity goes up as well. In other words, with the addition of hot wall length, both the conductive heat transfer and convective heat transfer rates improve, eventually leading to an increase of average $\mathrm{Nu}$. With the increase of hot wall length, the heat transfer rate grows more in case (a) than in case (b). The highest heat transfer rate is achieved in case (a), in the absence of a magnetic field and at a hot wall length of 0.6.

Fig. 18 shows the total generated entropies in cases (a) and (b) for different $\mathrm{Ha}$ and hot wall lengths for $R a=10^{5}, n=1, \gamma=45^{\circ}$. These graphs, which are very similar to the preceding ones, reveal that the amount of total generated entropy diminishes with the increase of $\mathrm{Ha}$. This is due to the decrease of vortex velocity and thus the reduction of velocity and temperature gradients. The diminishing of these two gradients leads to the respective decline of fluid friction entropy and thermal entropy, which eventually cause the reduction of total generated entropy. Also, with the increase in hot wall length, total entropy surges up as well. The underlying cause is the rise of velocity in the enclosure with the increase of hot wall length. The temperature field reveals that the temperature gradient, especially over the cold wall, goes up with the increase in hot wall length. This leads to the surge of thermal entropy. Moreover, the velocity gradient and, thus, the fluid friction entropy goes up with the rise of vortex velocity, thereby increasing the total entropy generated in the enclosure. The variations of generated entropies in both cases are very similar to each other. 
Fig. 19 displays the plotted $\mathrm{Be}$ obtained in cases (a) and (b) for different $\mathrm{Ha}$ and hot wall lengths for $R a=10^{5}, n=1, \gamma=45^{\circ}$. As it was mentioned, $B e$ indicates the share of thermal entropy in the total generated entropy. With the rise of $\mathrm{Ha}$, fluid velocity in the enclosure diminishes severely, due to the reduction of buoyancy force. This decreases the amount of fluid friction entropy. Also, with the escalation of $\mathrm{Ha}$, the temperature gradient falls slightly, thereby reducing the amount of thermal entropy. However, since the fluid friction entropy has declined substantially, with the increase of $\mathrm{Ha}$, the share of thermal entropy in the total generated entropy grows. So, $\mathrm{Be}$ goes up with the rise of $\mathrm{Ha}$. The variations of $B e$ with the increase of hot wall length, at various $H a$, show different trends. In both cases, in the absence of magnetic field, $B e$ rises with the increase of hot wall length. This is due to the elevation of temperature gradient and the increased share of thermal entropy in the total generated entropy. But at other $\mathrm{Ha}, \mathrm{Be}$ behaves differently for each case. The reason for this varying behavior is that, with the increase in the hot wall length, the velocity and temperature gradients go up and result in the growth of fluid friction entropy and thermal entropy; and in different cases, the share of thermal entropy can become more or less depending on the length of hot wall.

\subsection{Changing the tilt angle of enclosure}

Fig. 20 illustrates the contours of flow lines, isothermal lines and generated entropy lines in cases (a) and (b) for different enclosure tilt angles for $R a=10^{5}, H a=20, n=1$, $L=0.3$. In the flow field, we can see that the strongest vortex has occurred at the enclosure tilt angle of $90^{\circ}$. As it was previously mentioned, the rotation direction and the strength of a vortex depend on the Earth's magnetic field angle and acceleration of gravity. The angles of these two forces can strengthen or weaken a vortex and also determine its rotation direction. Hence, in an enclosure with a tilt angle of $0^{\circ}$, the formed 
vortexes rotate clockwise and in an enclosure tilted $90^{\circ}$, they have a counterclockwise rotation. In an enclosure tilted $45^{\circ}$, the vortex rotation direction in case (a) is opposite to that in case (b). As the fluid gets warmer near the hot wall of the enclosure, it becomes less dense and lighter in weight and, so, it moves upward. By reaching the upper section of the enclosure and contacting the cold wall, the fluid's temperature drops and it becomes denser. Thus, the fluid is pulled down by the force of gravity and it displaces the less heavy fluid below. In the meanwhile, the Lorentz force drives the fluid toward the right side of the enclosure. The sum of these motions creates a vortex in the enclosure. Whether the fluid is moved toward the right side of the enclosure in the enclosure's upper section or lower section determines the rotation direction of the formed vortex.

In the temperature field, the concentration of isothermal lines can be seen at different enclosure tilt angles, and this concentration does not change significantly in all the considered cases. The concentration of isothermal lines is more prevalent in the enclosure and gets closer to the cold wall only at the tilt angle of $90^{\circ}$. This is because in this case, the formed vortex is stronger and fluid circulates faster in the enclosure and causes the temperature gradient to rise in the colder region. The increase of temperature concentration near the cold wall means the improvement of heat transfer rate in this region; which can add to temperature difference in the enclosure and fortify the formed vortex.

In the contours of entropy lines, it is observed that the highest concentration of generated entropy lines has occurred at the enclosure tilt angle of $90^{\circ}$ in case (a) and tilt angle of $0^{\circ}$ in case (b). Because in these cases, better conditions have existed for heat transfer on these walls and, therefore, temperature gradients have become higher. With the rise of the temperature gradient, thermal entropy has also increased; so the concentration of generated entropies is higher in these regions. In general, in the areas where the hot wall 
runs into the insulated wall, because of the fluid having a higher temperature gradient, the amount of generated entropy is also greater relative to the other regions. In the corner regions of the enclosure, due to a less infiltration of fluid and the lower values of velocity and temperature gradients, the amount of generated entropy is also negligible; and this can be clearly seen in the entropy contours.

Fig. 21 displays the plotted local $N u$ on the hot wall of enclosure in cases (a) and (b) for different enclosure tilt angles and for constant values of $R a=10^{5}, H a=20, n=1$, $L=0.3$. As it is observed, with the increase of enclosure tilt angle, the maximum and minimum $N u$ on the hot wall diminish and the trend of local $N u$ becomes linear. At the tilt angle of $0^{\circ}$, at the beginning section of the hot wall, the local $N u$ is almost zero and at the end section of the wall it has a high value. In this case, there is a very little fluid circulation in the corner region of the enclosure, which causes $N u$ there to be near zero. However, at the end section of the hot wall, because of the large temperature gradient, the heat transfer rate is also high. With the increase in tilt angle and with the hot wall positioned vertically, the fluid is heated uniformly by the hot wall, and the uniform temperature gradient leads to a fixed heat transfer rate. Fig. 21(b) also shows the opposite of the previous trend. At the enclosure tilt angle of $90^{\circ}, \mathrm{Nu}$ has maximum and minimum values with large variations; while at lower tilt angles, the local $\mathrm{Nu}$ exhibits little variations along the hot wall. This case is opposite to the preceding case, and with the tilt angle reaching $0^{\circ}$, the hot wall becomes horizontal and fluid penetration into the corner zone diminishes, thereby reducing the rate of heat transfer in that region. The reason for the discrepancy in the two figures of (a) and (b) is the magnetic field. In the absence of the magnetic field, the two figures would have been identical; but the presence of a magnetic field has caused some differences in the local $N u$ in the two cases. 


\section{Conclusion}

In this paper, the natural convective characteristics of a non-Newtonian fluid inside a triangular enclosure, and subjected to a magnetic field, have been investigated. In addition to heat transfer, generated entropy and Bejan number have also been studied in this work. By varying the effective parameters of Rayleigh number, Hartmann number, power-law index, enclosure tilt angle and hot wall size, the following results have been obtained:

1- By raising the Rayleigh number from $10^{3}$ to $10^{5}$ in the non-Newtonian fluid, the heat transfer rate goes up by $71 \%$ in case (a) and $31 \%$ in case (b).

2- By raising the Rayleigh number the amount of thermal entropy increases by $80 \%$ (case a) and $88 \%$ (case b) in the Newtonian fluid, by $210 \%$ (case a) and $175 \%$ (case b) in the shear thickening non-Newtonian fluid

3- For a higher power-law index at Rayleigh number of $10^{5}$, the average Nusselt number decreases by $56 \%$ (case a) and $42 \%$ (case b) and the total generated entropy diminishes by $65 \%$ (case a) and $58 \%$ (case b).

4- In both cases of (a) and (b), the rise of Hartmann number leads to a $45 \%$ reduction in the heat transfer rate and the increase of Bejan number.

5- The increase of hot wall length leads to a higher heat transfer rate and total generated entropy.

\section{References}

[1] A.J. Chamkha, H. Al-Naser. Hydromagnetic double-diffusive convection in a rectangular enclosure with uniform side heat and mass fluxes and opposing temperature and concentration gradients. International Journal of Thermal Sciences. 41 (2002) 936-48.

[2] M.A. Ismael, A.J.J.J.o.p.m. Chamkha. Conjugate natural convection in a differentially heated composite enclosure filled with a nanofluid. 18 (2015). 
[3] A.J. Chamkha, I.V. Miroshnichenko, M.A.J.J.o.T.S. Sheremet, E. Applications. Numerical analysis of unsteady conjugate natural convection of hybrid water-based nanofluid in a semicircular cavity. 9 (2017) 041004.

[4] A. Chamkha. Natural convection of water-based nanofluids in a square enclosure with non-uniform heating of the bottom wall. (2013).

[5] S.M. Vahedi, A.H. Pordanjani, A. Raisi, A.J. Chamkha. Sensitivity analysis and optimization of MHD forced convection of a $\mathrm{Cu}$-water nanofluid flow past a wedge. The European Physical Journal Plus. 134 (2019) 124.

[6] W. Liu, A. Shahsavar, A. Barzinjy, A.A.A.A. Al-Rashed, M. Afrand. Natural convection and entropy generation of a nanofluid in two connected inclined triangular enclosures under magnetic field effects. International Communications in Heat and Mass Transfer. 108 (2019) 104309.

[7] M.A. Ismael, I. Pop, A.J. Chamkha. Mixed convection in a lid-driven square cavity with partial slip. International Journal of Thermal Sciences. 82 (2014) 47-61.

[8] R. Nasrin, M.A. Alim, A.J. Chamkha. Combined convection flow in triangular wavy chamber filled with water-CuO nanofluid: Effect of viscosity models. International Communications in Heat and Mass Transfer. 39 (2012) 1226-36.

[9] A.J. Chamkha, A. Al-Mudhaf. Double-diffusive natural convection in inclined porous cavities with various aspect ratios and temperature-dependent heat source or sink. Heat and Mass Transfer. 44 (2007) 679.

[10] A.J. Chamkha, M.A. Ismael. Conjugate Heat Transfer in a Porous Cavity Heated by a Triangular Thick Wall. Numerical Heat Transfer, Part A: Applications. 63 (2013) 14458.

[11] W.I. Liu, A.A.A.A. Al-Rashed, A.S. Alsagri, B. Mahmoudi, A. Shahsavar, M. Afrand. Laminar forced convection performance of non-Newtonian water-CNT/Fe $3 \mathrm{O}_{4}$ nano-fluid inside a minichannel hairpin heat exchanger: Effect of inlet temperature. Powder Technology. 354 (2019) 247-258.

[12] A. H. Pordanjani, A. Jahanbakhshi, A. A. Nadooshan, and M. Afrand, "Effect of two isothermal obstacles on the natural convection of nano-fluid in the presence of magnetic field inside an enclosure with sinusoidal wall temperature distribution," International Journal of Heat and Mass Transfer, vol. 121, pp. 565-578, 2018.

[13] C. Qi, L. Yang, G.J.N.r.l. Wang. Numerical study on convective heat transfer enhancement in horizontal rectangle enclosures filled with Ag-Ga nanofluid. 12 (2017) 326.

[14] C. Qi, G. Wang, L. Yang, Y. Wan, Z. Rao. Two-phase lattice Boltzmann simulation of the effects of base fluid and nanoparticle size on natural convection heat transfer of nanofluid. International Journal of Heat and Mass Transfer. 105 (2017) 664-72. 
[15] C. Qi, G. Wang, Y. Ma, L. Guo. Experimental Research on Stability and Natural Convection of TiO2-Water Nanofluid in Enclosures with Different Rotation Angles. Nanoscale Research Letters. 12 (2017) 396.

[16] C. Qi, L. Liang, Z. Rao. Study on the flow and heat transfer of liquid metal based nanofluid with different nanoparticle radiuses using two-phase lattice Boltzmann method. International Journal of Heat and Mass Transfer. 94 (2016) 316-26.

[17] E. Abu-Nada, A.J. Chamkha. Mixed convection flow of a nanofluid in a lid-driven cavity with a wavy wall. International Communications in Heat and Mass Transfer. 57 (2014) 36-47.

[18] S. Parvin, R. Nasrin, M.A. Alim, N.F. Hossain, A.J. Chamkha. Thermal conductivity variation on natural convection flow of water-alumina nanofluid in an annulus. International Journal of Heat and Mass Transfer. 55 (2012) 5268-74.

[19] A.J. Chamkha, H. Al-Naser. Hydromagnetic double-diffusive convection in a rectangular enclosure with opposing temperature and concentration gradients. International Journal of Heat and Mass Transfer. 45 (2002) 2465-83.

[20] A.A.A.A. Al-Rashed, A. Shahsavar, S. Entezari, M.A. Moghimi, S.A. Adio, T.K. Nguyen. Numerical investigation of non-Newtonian water-CMC/CuO nanofluid flow in an offset strip-fin microchannel heat sink: Thermal performance and thermodynamic considerations. Applied Thermal Engineering. 155 (2019) 247-258.

[21] A. Sarlak, A. Ahmadpour, and M. Hajmohammadi, "Thermal design improvement of a double-layered microchannel heat sink by using multi-walled carbon nanotube (MWCNT) nano-fluids with non-Newtonian viscosity," Applied Thermal Engineering, vol. 147, pp. 205-215, 2019.

[22] M. Vahabzadeh Bozorg and M. Siavashi, "Two-phase mixed convection heat transfer and entropy generation analysis of a non-Newtonian nano-fluid inside a cavity with internal rotating heater and cooler," International Journal of Mechanical Sciences, vol. 151, pp. 842-857, 2019/02.2019/01/

[23] C. Sasmal, A. K. Gupta, and R. P. Chhabra, "Natural convection heat transfer in a powerlaw fluid from a heated rotating cylinder in a square duct," International Journal of Heat and Mass Transfer, vol. 129, pp. 975-996, 2019/02/01/ 2019.

[24] R. R. Huilgol and G. H. R. Kefayati, "A particle distribution function approach to the equations of continuum mechanics in Cartesian, cylindrical and spherical coordinates: Newtonian and non-Newtonian fluids," Journal of Non-Newtonian Fluid Mechanics, vol. 251, pp. 119-131, 2018/01/01/2018.

[25] J. R. Reddy, K. A. Kumar, V. Sugunamma, and N. Sandeep, "Effect of cross diffusion on MHD non-Newtonian fluids flow past a stretching sheet with non-uniform heat 
source/sink: A comparative study," Alexandria engineering journal, vol. 57, pp. 1829$1838,2018$.

[26] H. Ragueb and K. Mansouri, "An analytical study of the periodic laminar forced convection of non-Newtonian nano-fluid flow inside an elliptical duct," International Journal of Heat and Mass Transfer,vol. 127, pp. 469-483, 2018/12/01/ 2018.

[27] A. Haque, A. K. Nayak, B. Soni, and M. Majhi, "Thermosolutal hydromagnetic convection of power law fluids in an enclosure with periodic active zones," International Journal of Heat and Mass Transfer, vol. 127 ,pp. 622-642, 2018/12/01/ 2018.

[28] P. Barnoon and D. Toghraie, "Numerical investigation of laminar flow and heat transfer of non-Newtonian nano-fluid within a porous medium," Powder Technology, vol. 325, pp. 78-91, 2018/02/01/ 2018.

[29] B. A. K. Naik and A. V. Vinod, "Heat transfer enhancement using non-Newtonian nanofluids in a shell and helical coil heat exchanger," Experimental Thermal and Fluid Science, vol. 90, pp. 132-142, 2018/01/01/ 2018.

[30] R. Kumar, S. Sood, S. A. Shehzad, and M. Sheikholeslami, "Radiative heat transfer study for flow of non-Newtonian nano-fluid past a Riga plate with variable thickness," Journal of Molecular Liquids, vol. 248, pp. 143-152, 2017/12/01/ 2017.

[31] O. Turan, A. Sachdeva, N. Chakraborty, and R. J. Poole, "Laminar natural convection of power-law fluids in a square enclosure with differentially heated side walls subjected to constant temperatures," Journal of Non-Newtonian Fluid Mechanics, vol. 166, pp. 10491063, 2011.

[32] M. Lamsaadi, M. Naimi, M. Hasnaoui, and M. Mamou, "Natural convection in a tilted rectangular slot containing non-Newtonian power-law fluids and subject to a longitudinal thermal gradient," Numerical Heat Transfer, Part A: Applications, vol. 50, pp. 561-583, 2006.

[33] G. B. Kim, J. M. Hyun, and H. S. Kwak, "Transient buoyant convection of a power-law non-Newtonian fluid in an enclosure," International Journal of Heat and Mass Transfer, vol. 46, pp. 3605-3617, 2003.

[34] A. I. Alsabery, A. J. Chamkha, H. Saleh, and I. Hashim, "Transient natural convective heat transfer in a trapezoidal cavity filled with non-Newtonian nano-fluid with sinusoidal boundary conditions on both sidewalls," Powder Technology, vol. 308, pp. 214-234, 2017/02/15/ 2017.

[35] G. R. Kefayati, "FDLBM simulation of entropy generation due to natural convection in an enclosure filled with non-Newtonian nano-fluid," Powder Technology, vol. 273, pp. 176-190, 2015/03/01/ 2015. 
[36] L. Mishra and R. P. Chhabra, "Natural convection in power-law fluids in a square enclosure from two differentially heated horizontal cylinders," Heat Transfer Engineering, vol. 39, pp. 819-842, 2018.

[37] G. R. Kefayati and H. Tang, "MHD thermosolutal natural convection and entropy generation of Carreau fluid in a heated enclosure with two inner circular cold cylinders, using LBM," International Journal of Heat and Mass Transfer, vol. 126, pp. 508-530, 2018/11/01/ 2018.

[38] P. Talebizadehsardari, A. Shahsavar, D. Toghraie, P. Barnoon. An experimental investigation for study the rheological behavior of water-carbon nanotube/magnetite nanofluid subjected to a magnetic field, Physica A. 534 (2019) 122129.

[39] J. Alsarraf, R. Rahmani, A. Shahsavar, M. Afrand, S. Wongwises. Effect of magnetic field on laminar forced convective heat transfer of $\mathrm{MWCNT}-\mathrm{Fe}_{3} \mathrm{O}_{4} /$ water hybrid nanofluid in a heated tube, Journal of Thermal Analysis and Calorimetry. 137 (2019) 1809-1825.

[40] W.I. Liu, J. Alsarraf, A. Shahsavar, M. Rostamzadeh, M. Afrand, T.K. Nguyen. Impact of oscillating magnetic field on the thermal-conductivity of water- $\mathrm{Fe}_{3} \mathrm{O}_{4}$ and waterFe3O4/CNT ferro-fluids: Experimental study, Journal of Magnetism and Magnetic Materials. 484 (2019) 258-265.

[41] A. Shahsavar, M. Bahiraei, R. Ansarian. Effect of line dipole magnetic field on entropy generation of $\mathrm{Mn}-\mathrm{Zn}$ ferrite ferrofluid flowing through a minichannel using two-phase mixture model, Powder Technology. 340 (2018) 370-379.

[42] G. R. Kefayati, "Mesoscopic simulation of magnetic field effect on natural convection of power-law fluids in a partially heated cavity," Chemical Engineering Research and Design, vol. 94, pp. 337-354, 2015/02/01/ 2015.

[43] S. Aghakhani, A. H. Pordanjani, A. Karimipour, A. Abdollahi, and M. Afrand, "Numerical Investigation of Heat Transfer in a Power-Law Non-Newtonian Fluid in a CShaped Cavity with Magnetic Field effect using Finite Difference Lattice Boltzmann Method," Computers \& Fluids, 2018.

[44] A. Bejan, Entropy generation through heat and fluid flow: Wiley, 1982.

[45] A. Bejan, "Second law analysis in heat transfer," Energy, vol. 5, pp. 720.1980 ,732-

[46] A. Shahsavar, M.H. Baseri, A.A.A.A. Al-Rashed, M. Afrand. Numerical investigation of forced convection heat transfer and flow irreversibility in a novel heatsink with helical microchannels working with biologically synthesized water-silver nano-fluid, International Communications in Heat and Mass Transfer. 108 (2019) 104324.

[47] A.A.A.A. Al-Rashed, A. Shahsavar, O. Rasooli, M.A. Moghimi, A. Karimipour, M.D. Tran. Numerical assessment into the hydrothermal and entropy generation characteristics 
of biological water-silver nano-fluid in a wavy walled microchannel heat sink, International Communications in Heat and Mass Transfer. 104 (2019) 118-126.

[48] A. Shahsavar, M. Moradi, M. Bahiraei. Heat transfer and entropy generation optimization for flow of a non-Newtonian hybrid nanofluid containing coated $\mathrm{CNT} / \mathrm{Fe} 3 \mathrm{O} 4$ nanoparticles in a concentric annulus, Journal of the Taiwan Institute of Chemical Engineers. 84 (2018) 149-161.

[49] G. Kefayati, "Heat transfer and entropy generation of natural convection on nonNewtonian nano-fluids in a porous cavity," Powder Technology, vol. 299, pp ,149-127. .2016

[50] M. Shojaeian and A. Koşar, "Convective heat transfer and entropy generation analysis on Newtonian and non-Newtonian fluid flows between parallel-plates under slip boundary conditions," International Journal of Heat and Mass Transfer, vol. 70, pp. 664673, 2014.

[51] S. Parvin, A.J. Chamkha. An analysis on free convection flow, heat transfer and entropy generation in an odd-shaped cavity filled with nanofluid. International Communications in Heat and Mass Transfer. 54 (2014) 8-17.

[52] M.A. Ismael, T. Armaghani, A.J. Chamkha. Conjugate heat transfer and entropy generation in a cavity filled with a nanofluid-saturated porous media and heated by a triangular solid. Journal of the Taiwan Institute of Chemical Engineers. 59 (2016) 138 51

[53] F. Selimefendigil, H.F. Öztop, A.J. Chamkha. MHD mixed convection and entropy generation of nanofluid filled lid driven cavity under the influence of inclined magnetic fields imposed to its upper and lower diagonal triangular domains. Journal of Magnetism and Magnetic Materials. 406 (2016) 266-81.

[54] A.H. Pordanjani, S. Aghakhani, A.A. Alnaqi, M. Afrand. Effect of alumina nano-powder on the convection and the entropy generation of water inside an inclined square cavity subjected to a magnetic field: Uniform and non-uniform temperature boundary conditions. International Journal of Mechanical Sciences. 152 (2019) 99-117.

[55] A. H. Pordanjani, S. Aghakhani, A. Karimipour, M. Afrand, and M. Goodarzi, "Investigation of free convection heat transfer and entropy generation of nano-fluid flow inside a cavity affected by magnetic field and thermal radiation," Journal of Thermal Analysis and Calorimetry, pp. 1-23

[56] M. Siavashi and A. Rostami, "Two-phase simulation of non-Newtonian nano-fluid natural convection in a circular annulus partially or completely filled with porous media," International Journal of Mechanical Sciences, vol. 133 ,pp. 689-703, 2017/11/01/ 2017. 
[57] Z. A. S. Raizah, A. M. Aly, and S. E. Ahmed, "Natural convection flow of a power-law non-Newtonian nano-fluid in inclined open shallow cavities filled with porous media," International Journal of Mechanical Sciences, vol. 140, pp. 376-393, 2018/05/01/ 2018.

[58] R. Benzi, S. Succi, and M. Vergassola, "The lattice Boltzmann equation: theory and applications," Physics Reports, vol. 222, pp. 145-197, 1992.

[59] S. Succi, The lattice Boltzmann equation: for fluid dynamics and beyond: Oxford university press, 2001.

[60] G. R. Kefayati, "Double-diffusive mixed convection of pseudoplastic fluids in a two sided lid-driven cavity using FDLBM," Journal of the Taiwan Institute of Chemical Engineers, vol. 45, pp. 2122-2139, 2014/0.2014/01/9

[61] G. R. Kefayati, "FDLBM simulation of mixed convection in a lid-driven cavity filled with non-Newtonian nano-fluid in the presence of magnetic field," International Journal of Thermal Sciences, vol. 95, pp. 29-46, 2015/09/01/ 2015.

[62] G.R. Kefayati, "FDLBM simulation of entropy generation in double diffusive natural convection of power-law fluids in an enclosure with Soret and Dufour effects," International Journal of Heat and Mass Transfer, vol. 89, pp. 267-290, 2015/10/01/ 2015 .

[63] S .Fu, R. So, and W. Leung, "Linearized-Boltzmann-type-equation-based finite difference method for thermal incompressible flow," Computers \& Fluids, vol. 69, pp. 67-80, 2012.

[64] S.-c. Fu, "Numerical simulation of blood flow in stenotic arteries," The Hong Kong Polytechnic University, 2011.

[65] S. C. Fu, R. M. C. So, and W. W. F. Leung, "Stochastic finite difference lattice Boltzmann method for steady incompressible viscous flows," Journal of Computational Physics, vol. 229, pp. 6084-6103, 2010/08/20/ 20.10

[66] L. Khezzar, D. Siginer, and I. Vinogradov, "Natural convection of power law fluids in inclined cavities," International Journal of Thermal Sciences, vol. 53, pp. 8-17, 2012/03/01/ 2012.

[67] R. R. Huilgol and G. H. R. Kefayati, "Natural convection problem in a Bingham fluid using the operator-splitting method," Journal of Non-Newtonian Fluid Mechanics, vol. 220, pp. 22-32, 2015/06/01/2015. 